\title{
Aspectos histológicos e imuno-histoquímicos das biópsias de medula óssea avaliadas no período de 2011 a 2013 em dois laboratórios gerais de anatomia patológica: dificuldades, facilidades e conclusões
} Histological and immunohistochemical aspects of marrow bone biopsies evaluated from 2013 in two general pathology laboratories: difficulties, advantages and conclusions

\begin{abstract}
RESUMO
Objetivo: As biópsias de medula óssea ganharam importância e passaram a colaborar, efetivamente, com as punções nesse órgão realizadas pelos hematologistas, após a melhoria significativa do uso da imuno-histoquímica em material parafinado. Esse exame requer informações clínicas e discussão dos casos entre patologistas e hematologistas, uma vez que as doenças hematopoéticas são um desafio para o patologista geral. Material e Método: O presente trabalho estudou 190 resultados de exames imuno-histoquímicos e os diagnósticos histológicos de todas as biópsias de medula óssea de dois laboratórios de patologia geral de Sorocaba (SP), no período de 2011 a 2013. Resultados e Discussão: A distribuição das frequências dos diagnósticos encontrados segue os padrões da literatura. No entanto, a maior dificuldade para chegar a esses diagnósticos foi a falta de dados clínicos, como faixa etária, informações clínicas/laboratoriais e hipóteses diagnósticas, que deveriam constar nas solicitações de exames e contribuiriam com a análise do médico patologista. O uso da imuno-histoquímica se confirmou como principal fator facilitador, imprescindível para o diagnóstico correto. A precisão do diagnóstico das doenças da medula óssea é importante para determinar o tratamento dos pacientes e para predizer fatores de melhor ou pior prognóstico. Conclusão: A associação entre o exame anatomopatológico e a imunohistoquímica, além dos dados clínicos e laboratoriais, deve estar presente na rotina de avaliação das biópsias de medula óssea. Palavras-chave: exame de medula óssea; biópsia por agulha; imuno-histoquímica; síndromes mielodisplásicas; leucemia.
\end{abstract}

\begin{abstract}
Objective: Bone marrow biopsies gained importance and began to collaborate effectively with the bone marrow punctures performed by hematologists, after the significant improvement in the use of immunohistochemistry in paraffinembedded material. This examination requires clinical discussion of cases between pathologists and hematologists, since hematopoietic diseases are a challenge for the general pathologist. Material and Method: This research studied 190 results of immunohistochemical tests and the histological diagnoses of all bone marrow biopsies performed in two general pathology laboratories of Sorocaba, state of São Paulo, Brazil, from 2011 to 2013. Results and Discussion: The distribution of the frequencies of diagnoses found follows the literature standards. However, the greatest difficulty to reach these diagnoses was the lack of clinical data, such as age, clinical/laboratory data and diagnostic hypotheses, which should be included in the test requests and contribute to the analysis of the pathologist. The use of immunohistochemistry was confirmed as the main facilitating factor, essential for the correct diagnosis. The accuracy of the diagnosis of bone marrow diseases is important to determine the treatment of patients and to predict better or worse prognosis factors. Conclusion: This study found that the association between the test pathology and immunohistochemistry, in addition to clinical and laboratory data, should be present in the routine evaluation of bone marrow biopsies.

Keywords: bone marrow examination; biopsy, needle; immunohistochemistry; myelodysplastic syndromes; leukemia.
\end{abstract}

${ }^{1}$ Pontifícia Universidade Católica de São Paulo (PUC-SP), Faculdade de Ciências Médicas e da Saúde - Sorocaba (SP), Brasil. Contato: douglaasalexandre18@gmail.com

Recebido em 29/02/2016. Aceito para publicação em 15/07/2016. 


\section{INTRODUÇÃO}

A biópsia de medula óssea (BMO) é parte integrante do estadiamento e seguimento de pacientes hematológicos e a sua avaliação oferece uma análise precisa da celularidade (relação tecido adiposo/tecido hematopoético), organização topográfica dos diferentes elementos celulares da medula óssea, alterações do estroma (fibrose reticulínica e colagênica), alterações morfológicas/topográficas dos elementos maduros da linhagem megacariocítica e lesões focais (metástases, infiltração por linfoma Hodgkin e linfoma não Hodgkin, granulomas). ${ }^{1}$

Apesar dos avanços importantes na área de patologia molecular, a morfologia da medula óssea continua a ser peça importante para identificar os vários subtipos de doenças mieloides. A possibilidade de realizar imuno-histoquímica, com marcadores celulares na biópsia de medula óssea, fixada em parafina, representa uma poderosa ferramenta de diagnóstico, de forma que seus resultados podem ser facilmente correlacionados com os obtidos por outras técnicas, como a citometria de fluxo e, acima de tudo, os achados clínicos. ${ }^{1-3}$

Com a introdução de anticorpos para os exames de imuno-histoquímica (IHQ), as indicações e a importância da BMO aumentaram e entraram na rotina da hematopatologia. Para a avaliação das BMO, é ideal contar com hematopatologista especialmente treinado, com a possibilidade de se relacionar com a imuno-histoquímica. ${ }^{2,3}$

Essa não é a realidade de laboratórios gerais de algumas cidades. Mesmo os hematopatologistas treinados precisam de informações clínicas hematológicas precisas, oportunidades de inter-relação com os hematologistas e, na maioria dos casos de estudo imuno-histoquímico, do material em questão. ${ }^{4}$ Esses são alguns dos desafios para o patologista, em laboratórios gerais. Em nosso meio, urge uma forte aproximação dos patologistas com os hematogistas - apesar das rotinas atarefadas de todos. Independentemente dos avanços tecnológicos, não se pode dispensar a necessidade de correlações clínicas para se chegar a um diagnóstico adequado., ${ }^{4,5}$

Este trabalho analisou os resultados dos casos de BMO nos últimos três anos, comparando os resultados da imuno -histoquímica com hematoxilina-eosina (também conhecida como H.E.) e colorações especiais, com comentários sobre a distribuição dos dados por idade, gênero, informações clínicas e hematológicas.

\section{MÉTODOS}

O projeto foi submetido ao Comitê de Ética em Pesquisa da Faculdade de Ciências Médicas e da Saúde da PUC-SP, sendo aprovado com autorização de dispensa do termo de consentimento por se tratar de estudo retrospectivo.
Foram levantados todos os exames de BMO de dois laboratórios (Laboratório de Patologia da Faculdade de Ciências Médicas e da Saúde de Sorocaba - FCMS/PUC-SP, e Laboratório de Patologia e Citologia de Sorocaba). Em ambos trabalham patologistas gerais que recebem todos os tipos de materiais anatomopatológicos para avaliação laboratorial.

Os casos apurados foram divididos por diagnósticos histológicos e imuno- histoquímicos, quando presentes. Os resultados foram interpretados por análise estatística descritiva simples. Os diagnósticos das reações de IHQ foram comparados com os diagnósticos histológicos e, por sua vez, ambos foram comparados com o quadro clínico de cada paciente hematológico, associado à faixa etária e ao gênero.

\section{RESULTADOS}

A Tabela 1 mostra o gênero e a faixa etária da casuística avaliada neste trabalho. A Tabela 2 correlaciona o número de pacientes às doenças hematológicas encontradas na pesquisa.

Tabela 2. Distribuição dos diagnósticos patológicos.

\begin{tabular}{lc} 
Diagnóstico & $\begin{array}{c}\text { Número de } \\
\text { pacientes }\end{array}$ \\
\hline Normalidade & 51 \\
\hline Hiperplasia & 36 \\
\hline Hipoplasia & 26 \\
\hline Neoplasias linfoproliferativas & 23 \\
\hline Síndromes mieloproliferativas & 17 \\
LMC & 7 \\
Policitemia vera & 3 \\
Mielofibrose primária & 2 \\
Não especificado & 5 \\
\hline Síndromes mielodisplásicas & 11 \\
\hline Plasmocitoma/mieloma múltiplo & 11 \\
\hline Metástases & 5 \\
CA pouco diferenciado & 2 \\
CA de mama & 1 \\
\hline Adenocarcinoma & 1 \\
\hline Neuroblastoma & 1 \\
\hline Aplasia & 3 \\
\hline Anemia megaloblástica & 1 \\
\hline Inconclusivos & 6 \\
\hline
\end{tabular}

Tabela 1. Distribuição dos pacientes por genero e faixa etária.

\begin{tabular}{lcccccc} 
Gênero/Idade & $\mathbf{0} \mathbf{a} \mathbf{2 0}$ anos & $\mathbf{2 1} \mathbf{a} \mathbf{4 0}$ anos & $\mathbf{4 1} \mathbf{~ a ~} \mathbf{6 0}$ anos & $\mathbf{6 1}$ ou mais & Não informado & Total \\
\hline Masculino & 10 & 9 & 23 & 28 & 22 & 92 \\
\hline Feminino & 6 & 15 & 25 & 25 & 27 & 98 \\
\hline Total & 16 & 24 & 48 & 53 & 49 & 190 \\
\hline
\end{tabular}


Como é possível visualizar na Tabela 1 , foram analisados 190 resultados de exames anatomopatológicos de medula óssea, sendo 51,6\% $(\mathrm{n}=98)$ de pacientes do sexo feminino e $48,4 \%(\mathrm{n}=92)$ do sexo masculino. Apenas 141 exames continham a idade dos pacientes e, desses, 71,6\% $(\mathrm{n}=101)$ pertencem à faixa etária acima de 41 anos. Não se obteve informações da faixa etária em 25,7\% ( $\mathrm{n}=49)$ dos exames. Apenas 22 exames não continham análise imuno-histoquímica.

O diagnóstico de normalidade esteve presente em 26,8\% $(\mathrm{n}=51)$ dos exames (Tabela 2). Alterações apenas de celularidade (hiperplasia, hipoplasia e aplasia) corresponderam a $34,2 \%(n=65)$ dos diagnósticos e 3,1\% ( $n=6)$ dos exames permaneceram inconclusivos. As doenças mielodisplásicas e mieloproliferativas representaram, em conjunto, $15,3 \%$ ( $\mathrm{n}=$ 28) do total de exames, enquanto as neoplasias linfoproliferatifvas corresponderam a $12,1 \%(n=23)$. Os resultados contidos na Tabela 2 mostram com mais especificidade os diagnósticos obtidos, cuja precisão dependeu fundamentalmente das reações de imuno-histoquímica.

A Tabela 3 relaciona as informações contidas em 101 solicitações de BMO do total dos casos analisados com os respectivos diagnósticos obtidos. Em 30,8\% das solicitações não havia informações clínicas ou hipótese diagnóstica e, dessas, $45,2 \%$ obtiveram diagnóstico de malignidade. Em 34,5\% das subscrições havia hipótese diagnóstica e 34,7\% apresentavam alguma informação clínica.

O estadiamento de linfoma não Hodgkin foi o motivo de biópsia mais citado nas solicitações (14,9\%), sendo que $40 \%$ estavam dentro dos padrões da normalidade. A síndrome mielodisplásica (SMD) foi citada como hipótese diagnóstica em
9,8\% das solicitações e apenas 30\% dos casos se confirmaram pelos exames anatomopatológicos e imuno-histoquímicos. Todos os casos em que a neutropenia crônica foi citada como motivo da BMO obtiveram diagnóstico de hipoplasia medular. As neoplasias linfoproliferativas foram indicadas como hipótese diagnóstica em 5,9\% das solicitações e o diagnóstico se confirmou após o exame histológico em todos esses casos.

A distribuição de diagnósticos de acordo com sexo e faixa etária dos pacientes está retratada na Tabela 4. Nota-se que os diagnósticos de malignidade se mostram significativamente mais prevalentes em pacientes acima de 41 anos. Os resultados compatíveis com neoplasias linfoproliferativas e metástases foram, respectivamente, duas e quatro vezes mais frequentes no sexo feminino. Já entre os pacientes com diagnóstico de síndromes mieloproliferativas (SMP), 64,7\% eram do sexo masculino.

\section{DISCUSSÃO}

Os exames anatomopatológicos e de imuno-histoquímica são indispensáveis para o diagnóstico preciso das alterações da medula óssea. No entanto, faz-se necessário correlacionar as alterações morfológicas com as informações clínicas dos pacientes para que o diagnóstico seja conclusivo. ${ }^{1-8}$

Em poucas subscrições foram citadas as informações clínicas ou laboratoriais dos pacientes; o mesmo se observou quanto às hipóteses diagnósticas. Chama a atenção, ainda, o fato de $25,7 \%$ dos exames não informar as idades dos pacientes.

A ausência dessas informações pode dificultar a interpretação dos casos pelo patologista. Além disso, a falta desses dados não é justificável, uma vez que não há dificuldade em

Tabela 3. Informações contidas em solicitações de exames e confirmação diagnóstica.

\begin{tabular}{|c|c|c|c|c|c|c|c|c|c|c|c|}
\hline \multirow{2}{*}{$\begin{array}{l}\text { Pedido/Solicitação } \\
\text { Hipótese diagnóstica ou } \\
\text { Informação Clínica }\end{array}$} & \multicolumn{11}{|c|}{ Resultado de AP/IHQ } \\
\hline & Normal & Hiper & Hipo & SMD & SMP & LNH & NLP & MM & Aplasia & Metástase & Total \\
\hline Ausentes & 2 & 8 & 5 & 2 & 7 & 3 & 2 & - & 1 & 1 & 31 \\
\hline Estadiamento de LNH & 6 & 2 & 3 & - & - & 3 & 1 & - & - & - & 15 \\
\hline Síndrome nielodisplásica & 3 & - & - & 3 & - & - & 2 & - & 2 & - & 10 \\
\hline Anemia crônica & 1 & - & - & - & 3 & - & - & 3 & - & - & 7 \\
\hline Mieloma múltiplo & 2 & - & - & - & - & 2 & - & 3 & - & - & 7 \\
\hline Síndrome mieloproliferativa & 1 & - & - & - & 5 & - & - & - & - & - & 6 \\
\hline Doença linfoproliferativa & - & - & - & - & - & - & 6 & - & - & - & 6 \\
\hline Metástases & 1 & - & - & - & - & - & - & - & - & 3 & 4 \\
\hline Leucopenia crônica & 3 & - & 1 & - & - & - & - & - & - & - & 4 \\
\hline Neutropenia crônica & - & - & 4 & - & - & - & - & - & - & - & 4 \\
\hline Plaquetopenia & 1 & 1 & - & - & 1 & - & - & - & - & - & 3 \\
\hline Recidiva de LNH & - & - & 1 & - & - & - & - & 1 & - & - & 2 \\
\hline Pancitopenia & - & - & - & 1 & - & 1 & - & - & - & - & 2 \\
\hline
\end{tabular}

AP: Anatomopatológico. IHQ: Imuno-histoquímica. Hiper: hiperplasia. Hipo: hipoplasia. SMD: Síndrome mielodisplásica. SMP: Síndrome mieloproliferativa. LNH: Linfoma não Hodgkin. MM: Mieloma múltiplo. 
fornecê-los ao patologista, por meio do preenchimento coeso e completo das solicitações dos exames.

A associação entre os exames anatomopatológicos e as imuno-histoquímicas mostrou-se fundamental principalmente em três aspectos: na confirmação ou afastamento de malignidade; no diagnóstico diferencial das mielodisplasias, neoplasias mieloproliferativas e linfoproliferativas; e, ainda, na determinação das infiltrações e metástases.

Nas SMD (confirmadas em 5,8\% $(\mathrm{n}=11)$ dos casos), por exemplo, observa-se que a IHQ tem duas utilidades principais: caracterizar as três linhagens mieloides, auxiliando na identificação de sua desorganização arquitetural e formas atípicas/displásicas; e na diferenciação da forma hipocelular da SMD com a anemia aplástica. Observa-se, no entanto, que os laudos imuno-histoquímicos com diagnóstico de SMD sugerem, em sua totalidade, a análise por citometria de fluxo para determinar subtipos histológicos.

No contexto das síndromes mieloproliferativas, presentes em 9\% $(\mathrm{n}=17)$ dos exames analisados, foi possível determinar (com auxílio da IHQ) os diagnósticos de leucemia mieloide crônica (LMC), policitemia vera (PV) e mielofibrose primária (MF). Ainda assim, cinco casos permaneceram não especificados mesmo utilizando os recursos supracitados.
Os casos inconclusivos, portanto, necessitam de um painel maior de marcadores celulares na reação de IHQ, além de correlações clínicas para fechar o diagnóstico. A imuno-histoquímica, nesses casos, também foi importante para estimar o número de blastos e confirmar ou afastar a suspeita de evolução para uma fase acelerada ou transformação aguda das neoplasias mieloproliferativas.

As doenças linfoproliferativas com diferenciação plasmocitária e pico monoclonal geralmente são causas de dificuldade diagnóstica no estudo da medula óssea. O diagnóstico diferencial é amplo e, por esse motivo, a imunofenotipagem mostrou-se indispensável.

A classificação morfológica e imuno-histoquímica precisa permitiu a separação das infiltrações de medula óssea entre os tipos de linfoma não Hodgkin e neoplasias imaturas, como observado em 7,9\% $(\mathrm{n}=15)$ dos exames analisados. Essa diferenciação mostra-se essencial por constituir um fator determinante de melhor ou pior prognóstico para os pacientes, sendo, também, decisiva nas novas perspectivas terapêuticas.

As principais neoplasias responsáveis pelas metástases em medula óssea encontradas no presente estudo foram o neuroblastoma e o câncer de mama, semelhante ao que ocorre na literatura.

Tabela 4. Distribuição de diagnósticos de acordo com o sexo e a faixa etária dos pacientes.

\begin{tabular}{|c|c|c|c|c|c|c|c|}
\hline Diagnóstico & Sexo & $\begin{array}{c}0-20 \\
\text { Anos }\end{array}$ & $\begin{array}{l}21-40 \\
\text { Anos }\end{array}$ & $\begin{array}{l}41-60 \\
\text { Anos }\end{array}$ & $\begin{array}{l}\geq 61 \\
\text { Anos }\end{array}$ & $\begin{array}{c}\text { Não } \\
\text { informado }\end{array}$ & Total \\
\hline Normalidade & $\begin{array}{l}\text { M: } 29 \\
\text { F: } 22\end{array}$ & 2 & 9 & 11 & 18 & 11 & 51 \\
\hline Hiperplasia & $\begin{array}{l}\text { M: } 16 \\
\text { F: } 20\end{array}$ & 0 & 6 & 12 & 8 & 10 & 36 \\
\hline Hipoplasia & $\begin{array}{l}\text { M: } 15 \\
\text { F: } 11\end{array}$ & 8 & 3 & 4 & 5 & 6 & 26 \\
\hline $\begin{array}{l}\text { Síndromes } \\
\text { mieloproliferativas }\end{array}$ & $\begin{array}{l}\text { M: } 11 \\
\text { F: } 6\end{array}$ & 3 & 2 & 3 & 3 & 6 & 17 \\
\hline Síndromes mielodisplásicas & $\begin{array}{l}\text { M: } 5 \\
\text { F: } 6\end{array}$ & 0 & 1 & 5 & 4 & 1 & 11 \\
\hline Neoplasias linfoproliferativas & $\begin{array}{l}\text { M: } 7 \\
\text { F: } 16\end{array}$ & 0 & 3 & 6 & 7 & 7 & 23 \\
\hline Plasmocitoma (mieloma múltiplo) & $\begin{array}{l}\text { M: } 4 \\
\text { F: } 7\end{array}$ & 0 & 0 & 2 & 6 & 3 & 11 \\
\hline Metástases & $\begin{array}{l}\text { M: } 1 \\
\text { F: } 4\end{array}$ & 1 & 0 & 2 & 0 & 2 & 5 \\
\hline Aplasia & $\begin{array}{l}\text { M: } 3 \\
\text { F: } 0\end{array}$ & 2 & 0 & 0 & 0 & 1 & 3 \\
\hline Anemia megaloblástica & $\begin{array}{l}\text { M: } 0 \\
\text { F: } 1\end{array}$ & 0 & 0 & 0 & 0 & 1 & 1 \\
\hline Inconclusivo & $\begin{array}{l}\text { M: } 1 \\
\text { F: } 5\end{array}$ & 0 & 0 & 3 & 2 & 1 & 6 \\
\hline Total & $\begin{array}{l}\text { M: } 92 \\
\text { F: } 98\end{array}$ & 16 & 24 & 48 & 53 & 49 & 190 \\
\hline
\end{tabular}




\section{CONCLUSÕES}

A principal dificuldade encontrada nos exames anatomopatológicos e imuno-histoquímicos de medula óssea consiste na falta de dados, como faixa etária, informações clínicas/laboratoriais e hipóteses diagnósticas que, juntas, contribuiriam para a análise do médico patologista. $\mathrm{O}$ preenchimento completo das solicitações de exames, portanto, consiste em fator facilitador.

A distribuição de frequências dos diagnósticos encontrados, relacionados ao sexo e à faixa etária, seguiu o padrão da literatura. Os principais foram: alterações isoladas de celularidade (62 casos), SMP (17 casos), doenças linfoproliferativas (23 casos), SMD (11 casos), mieloma múltiplo (11 casos) e metástases ( 5 casos).

O diagnóstico das SMP foi mais frequente em homens, assim como consta na literatura. A prevalência do diagnóstico de metástases e doenças linfoproliferativas foi substancialmente maior em pessoas do sexo feminino, nesse estudo.

A IHQ apresentou aspectos positivos em todos os casos, constituindo um fator facilitador e imprescindível para o diagnóstico correto. Suas principais utilidades foram: confirmação do diagnóstico anatomopatológico, diagnóstico diferencial das SMD/SMP, identificação de infiltrações e metástases e determinação de subtipos celulares de neoplasias.

Os casos inconclusivos (seis casos) podem necessitar de recursos técnicos adicionais, como a citometria de fluxo e a hibridizaçao in situ fluorescente (também chamada de FISH), além de correlações clínicas para a conclusão do diagnóstico.

A precisão do diagnóstico das doenças da medula óssea é importante para determinar o tratamento dos pacientes e para predizer fatores de melhor ou pior prognóstico. Por isso, a associação entre o exame anatomo patológico (conhecido como EAP) e a IHQ, além dos dados clínicos e laboratoriais, deve estar presente na rotina de avaliação das biópsias de medula óssea.

\section{AGRADECIMENTOS}

Agradecemos ao APC Laboratório de Anatomia Patológica e Citologia S/A, ao Laboratório de Anatomia Patológica e Citologia da FOSP, ao Laboratório Stecca de Patologia e Citologia de Sorocaba e ao Laboratório de Anatomia Patológica da FCMS, Sorocaba - PUC-SP.

\section{REFERÊNCIAS}

1. Hoffbrand AV, Moss PAH. Fundamentos em hematologia. 6. ed. Porto Alegre: Artmed; 2013.

2. OraziA.Histopathologyinthediagnosisandclassification of acutemyeloid leukemia, myelodysplastic syndromes, and myelodysplastic/myeloproliferative diseases. Pathobiology. 2007;74(2):97-114.

3. Dunphy CH, O'Malley DP, Perkins SL, Chang CC. Analysis of immunohistochemical markers in bone marrow sections to evaluate for myelodysplastic syndromes and acute myeloid leukemias. Appl Immunohistochem Mol Morphol. 2007;15(2):154-9.

4. Fend F, Kremer M. Diagnosis and classification of malignant lymphoma and related entities in the bone marrow trephine biopsy. Pathobiology. 2007;74(2):133-43.

5. Orazi A, Chiu R, O'Malley DP, Czader M, Allen SL, An C, et al. Chronic myelomonocytic leukemia: the role of bone marrow biopsy immunohistology. Mod Pathol. 2006;19:1536-45.

6. Horny HP, Sotlar K, Valent P. Diagnostic value of histology and immunohistochemistry in myelodysplastic syndromes. Leuk Res. 2007;31(12):1609-16.

7. Kremer M, Quintanilla-Martínez L, Nährig J, von Schilling C, Fend F. Immunohistochemistry in bone marrow pathology: a useful adjunct for morphologic diagnosis. Virchows Arch. 2005;447(6):920-37.

8. Fend F, Tzankov A, Bink K, Seidl S, QuintanillaMartinez L, Kremer M, et al. Modern techniques for the diagnostic evaluation of the trephine bone marrow biopsy: Methodological aspects and applications. Prog Histochem Cytochem. 2008;42(4):203-52. 